OPEN ACCESS

Edited by:

George Weinstock

Jackson Laboratory for Genomic

Medicine, United States

Reviewed by:

Yingli He,

Xi'an Jiaotong University, China

Yu-Chen Fan,

Shandong University, China

${ }^{*}$ Correspondence:

Yimin Zhang

1307020@zju.edu.cn

tThese authors have contributed equally to this work

Specialty section:

This article was submitted to Infectious Diseases - Surveillance,

Prevention and Treatment

a section of the journal

Frontiers in Medicine

Received: 08 November 2020

Accepted: 20 January 2021

Published: 24 February 2021

Citation:

Jin Y, Wang Z, Zhu C, Yang Q, Lu Y, $Y u X$, Hong $B$, Wang $X$ and Zhang $Y$

(2021) Case Report: Proven Diagnosis of Culture-Negative Chronic Disseminated Candidiasis in a Patient

Suffering From Hematological

Malignancy: Combined Application of mNGS and CFW Staining.

Front. Med. 8:627166.

doi: 10.3389/fmed.2021.627166

\section{Case Report: Proven Diagnosis of Culture-Negative Chronic Disseminated Candidiasis in a Patient Suffering From Hematological Malignancy: Combined Application of mNGS and CFW Staining}

\author{
Yanqi Jin ${ }^{1+}$, Zhouhan Wang ${ }^{1 \dagger}$, Chunxia Zhu ${ }^{1}$, Qing Yang ${ }^{2}$, Yingfeng $\mathrm{Lu}^{1}$, Xiaopeng Yu ${ }^{1}$, \\ Bao Hong ${ }^{3}$, Xiaojing Wang ${ }^{3}$ and Yimin Zhang ${ }^{1,3 *}$
}

\begin{abstract}
${ }^{1}$ State Key Laboratory for Diagnosis and Treatment of Infectious Diseases, National Clinical Research Center for Infectious Diseases, Collaborative Innovation Center for Diagnosis and Treatment of Infectious Diseases, The First Affiliated Hospital, College of Medicine, Zhejiang University, Hangzhou, China, ${ }^{2}$ Laboratory Department, The First Affiliated Hospital, College of Medicine, Zhejiang University, Hangzhou, China, ${ }^{3}$ Department of Infectious Diseases of Haining Campus, The First Affiliated Hospital, College of Medicine, Zhejiang University, Haining, China
\end{abstract}

Chronic disseminated candidiasis (CDC) is a severe complication with high morbidity and mortality in patients with hematological malignancies who have undergone chemotherapy. Blood or sterile liver biopsy cultures are negative due to recurrent empirical antifungal therapy. With the escalating resistance to azole-based antifungal drugs in infection by Candida species, pathogen identification is becoming increasingly important for determining definitive diagnosis and treatment strategy. In this case report, we present, for the first time, diagnostic confirmation of a culture-negative CDC case with Candida tropicalis infection using a combination of metagenomics next-generation sequencing and calcofluor white staining.

Keywords: chronic disseminated candidiasis, metagenomics next-generation sequencing, Candida tropicalis, calcofluor white staining, hematological malignancies, proven diagnosis

\section{INTRODUCTION}

Chronic disseminated candidiasis (CDC; also known as "hepatosplenic candidiasis") is a unique manifestation of invasive candidiasis. CDC carries significant morbidity and mortality in patients with hematological malignancies who have undergone chemotherapy (1-3). The previous diagnostic criteria were positive histology plus culture evidence for Candida species from blood or a sterile liver biopsy $(4,5)$. The chance of positive findings in blood cultures with an onset of the clinical and radiological picture suggestive of CDC is poor (3). The prevalence of a proven diagnosis is quite low due to the difficulty of liver puncture and recurrent use of empirical antifungal prophylaxis after chemotherapy.

Development of metagenomics next-generation sequencing (mNGS) has enabled identification of causative pathogens in infectious diseases (6) and has been applied for the diagnosis of CDC. We report a culture-negative CDC case with Candida tropicalis (C. tropicalis) infection diagnosed by mNGS and calcofluor white (CFW) staining. 
Description of this case study was approved (2020IIT 593) by the Ethics Committee of the First Affiliated Hospital of Zhejiang University (Hangzhou, China) and by the patient.

\section{CASE PRESENTATION}

A 62-year-old woman with acute myeloid leukemia-M2 received remission-induction chemotherapy (day-0). An outline of the following episodes is described in Figure 1. From day0 , recurrent fever occurred (Figure 2). Empirical treatment was undertaken with broad-spectrum antibiotics. The patient experienced neutropenia from day-2 to day-19. Antifungal prophylaxis was not employed until day-18, and yielded a positive result for the $(1,3)-\beta$-D-glucan $(B D G)$ test $(470 \mathrm{pg} / \mathrm{mL})$. At that time, the results of a series of blood cultures were negative from day-16 to day-18 (Figure 1). Computed tomography (CT) of the abdomen showed multiple low-density lesions in the liver, spleen, and kidneys in day-28.

Caspofungin (CAP) from day-18 to day-28 and voriconazole (VRC) from day-28 to day-38 were used as antifungal therapy after a positive result for the BDG test on day-18 (Figure 1). However, the recurrent fever persisted without remission for the lesions on the liver, spleen, and kidneys even after recovery from neutropenia.

Contrast-enhanced CT of the abdomen showed multiple lesions with hypodensity in the liver, spleen, and kidneys as characterized by marked rim enhancement (Figure 1A). Ultrasound of the abdomen, enhanced magnetic resonance imaging (MRI) of the abdomen, ${ }^{18}$ F-fluorodeoxyglucosepositron emission tomography/computed tomography, and contrast-enhanced ultrasound confirmed the result of contrastenhanced CT of the abdomen (Figure 3). While a follow-up BDG test produced a negative result on day- 43 and on several subsequent occasions (Figure 1). Expectedly, several cultures of blood and feces on different days failed to grow any pathogen.

On day-54, ultrasound-mediated liver puncture and biopsy was undertaken (Figure 2). A specimen was obtained between the boundary of lesions and normal liver tissues. Histology showed very few epithelioid cells, possibly a tumor, but no evidence of fungal infection. The antifungal agent was changed from VRC to CAP due to suspicion of CDC (Figure 2). However, her condition did not improve significantly, and recurrent fever persisted (Figure 2).

Another liver biopsy was done 1-week later (day-62). The puncture needle passed through the entire lesion to reach its center, and tissues were sampled. Histology revealed granulomatous inflammation with necrosis and no evidence of malignancy (Figures 1B,C). Immunohistochemistry showed positive in TB (FISH) (only one found) but negative in fungal (FISH). The serum T-SPOT ${ }^{\circledR}$.TB interferon- $\gamma$ release assay for active tuberculosis was negative. No pathogen was cultured from the two liver-biopsy tissues.

The samples from the second liver puncture were tested using mNGS (Figure 1). The results showed 55 total reads of C. tropicalis, accounting for $98.21 \%$ total fungal reads which was sentenced as responsible pathogen in accordance with other

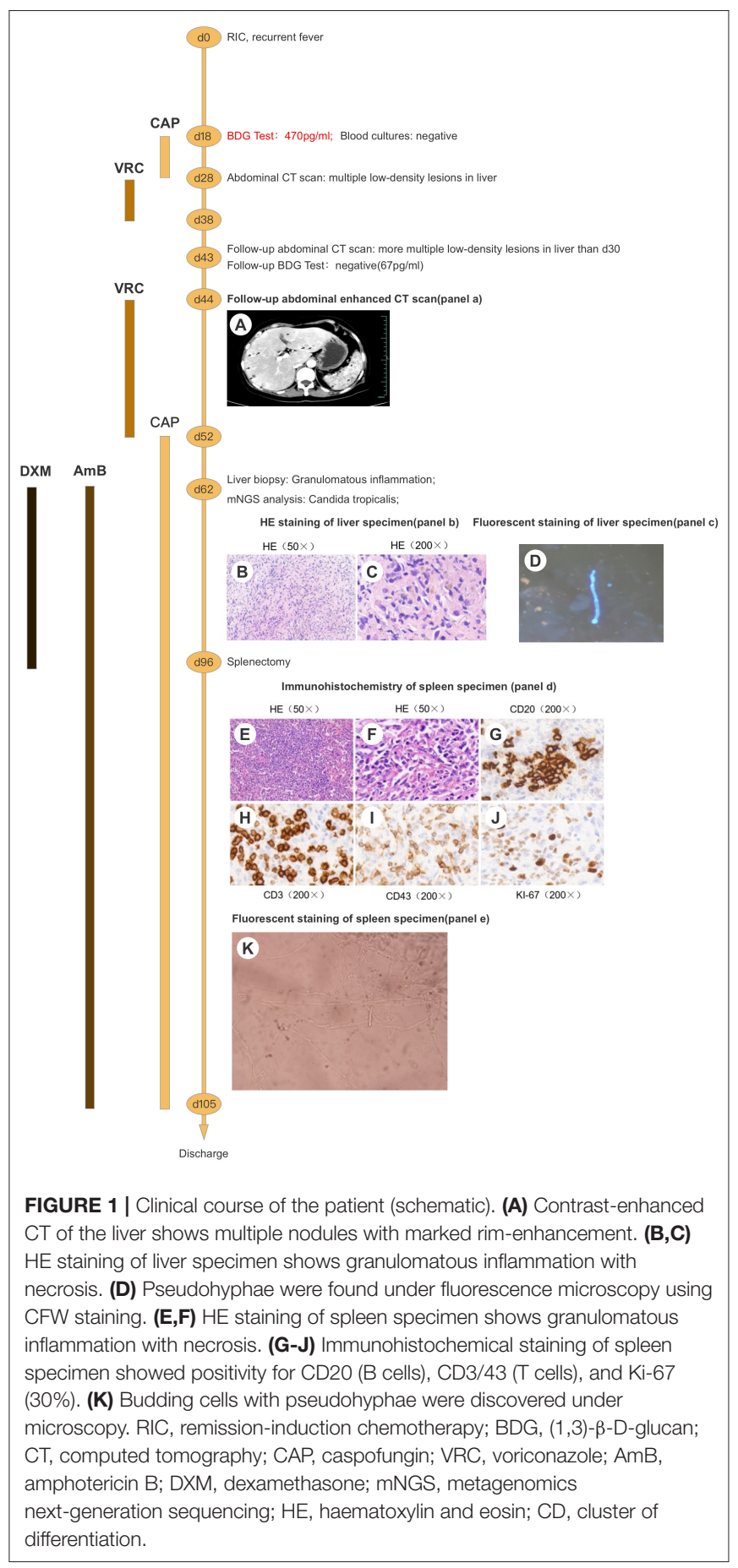

clinical evidences. Interestingly, pseudohyphae were found under fluorescence microscopy using CFW staining (Figure 1D) to confirm the mNGS result. After consideration of detection by mNGS and the microscopic appearance, we decided to add the antifungal agent (amphotericin B (AmB), $25 \mathrm{mg}$ per day, i.v.) and adjuvant corticosteroid (dexamethasone (DXM), $7.5 \mathrm{mg}$ per day, i.v.) for further treatment (Figure 2). After adjusting the 


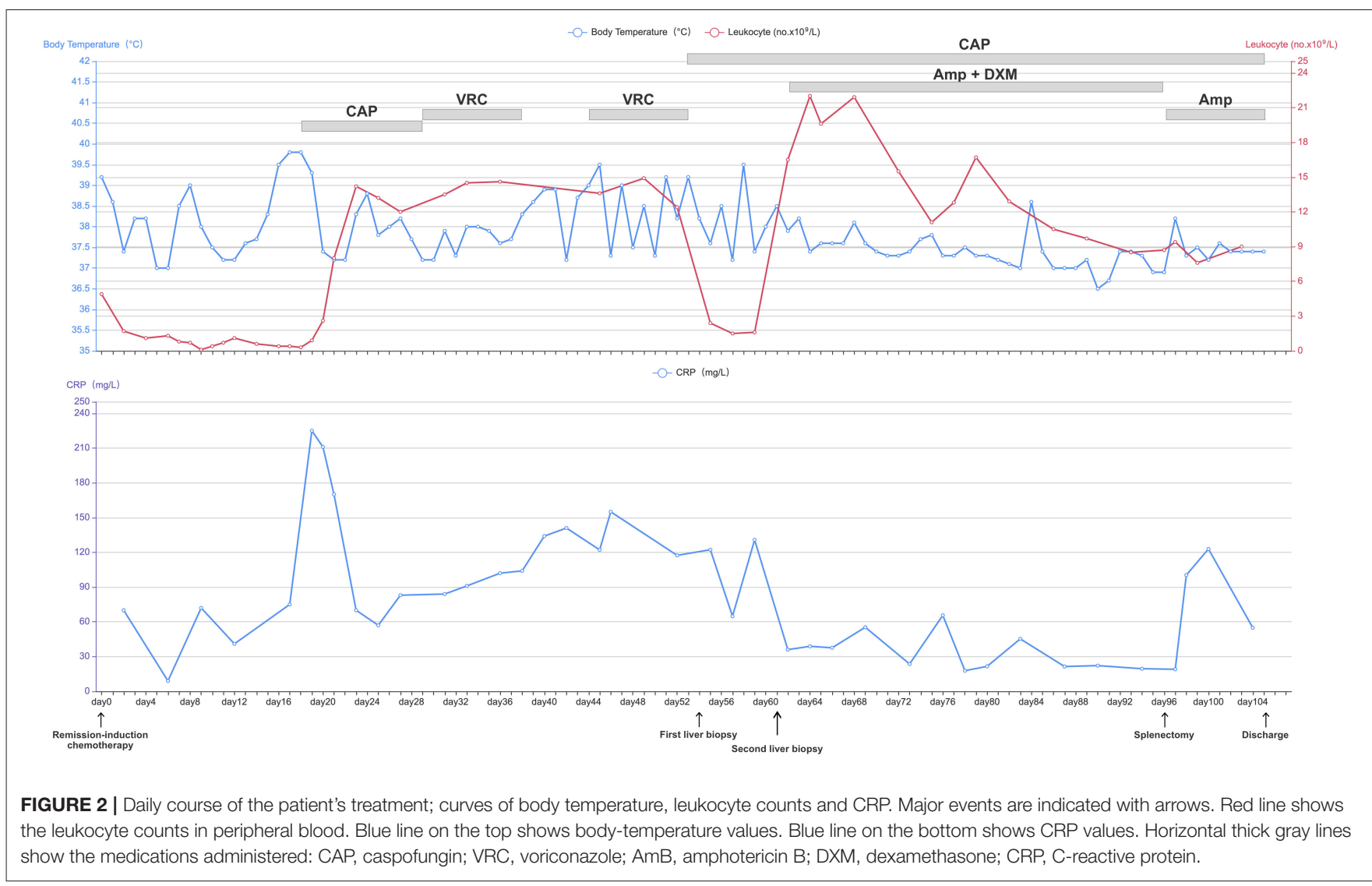

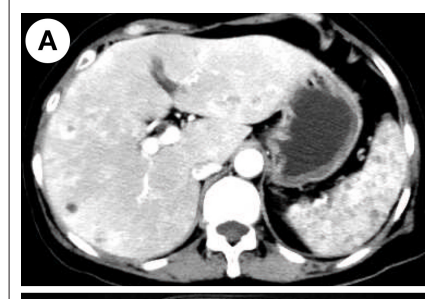
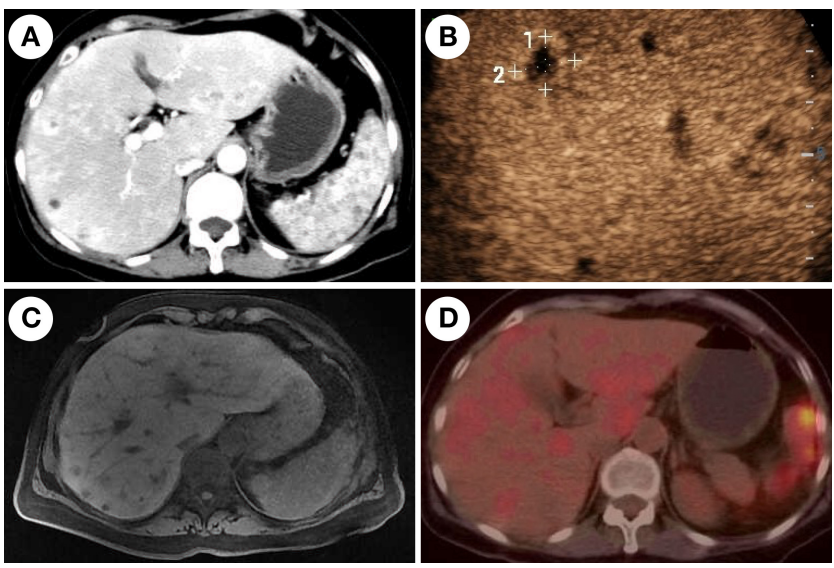

FIGURE 3 | Imaging features of chronic disseminated candidiasis. (A) Contrast-enhanced computed tomography of the abdomen shows multiple nodules with marked rim-enhancement. (B) Contrast-enhanced ultrasound shows multiple hypo-echoic lesions in the liver. (C) Magnetic resonance imaging shows multiple-sized circular abnormal signal lesions in the liver and spleen. (D) ${ }^{18} \mathrm{~F}$-fluorodeoxyglucose-positron emission tomography/computed tomography $\left({ }^{18} \mathrm{~F}-\mathrm{FDG}-\mathrm{PET} / \mathrm{CT}\right)$ shows multiple lesions in the liver and spleen with increased metabolism of FDG.

medication regimen, the body temperature dropped to a normal level (Figure 2).

$\mathrm{CAP}+\mathrm{AmB}+\mathrm{DXM}$ therapy was continued for $>6$ weeks while patient had a normal temperature but improvement in imaging features (lesions on the liver, spleen, and kidneys) was not observed. On day-96, splenectomy was undertaken after consultation with the multidisciplinary team (Figure 1). Multiple dense abscesses were found in the entire spleen, and granulomatous inflammation with necrosis was observed in splenic tissue under microscopy (Figures 1E,F). Budding cells with pseudohyphae consistent with infection by Candida species were observed under microscopy (Figure 1K). Immunohistochemistry showed leukocyte common antigen and positivity for cluster of differentiation (CD)3/43 ( $\mathrm{T}$ cells), CD20 (B cells), and Ki-67 (30\%), whereas a methenamine silver stain was negative (Figures 1G-J). After splenectomy, the patient symptoms were alleviated with a decrease of CRP level and white blood cell count (Figure 2). Her body temperature remained normal and we discontinued use of DXM (Figure 2). On day-105, the patient was discharged with continuing antifungal therapy of CAP and AmB. Cytological examination of the bone marrow showed complete remission. The patient was transferred to local hospital to continue antifungal therapy and was suggested to follow-up with CT scan every 2 months.

\section{DISCUSSION}

The high morbidity and mortality in CDC patients necessitates rapid diagnostic. Few cases are confirmed by culture, this is supported by a retrospective single-center study from 2008 to 2013 (7). It showed very low positive results from both of blood 
culture ( $\sim 20 \%)$ and liver biopsy(10-20\%) culture from patients undergoing chemotherapy and administrated with empirical antifungal therapy (7-9).

Cultures are limited further by slow turnaround times (typically requiring 2-3 days for growth to be evident), and the fact that they often turn positive late in the course of infection (10). Hence, according to previous "gold standard" diagnostic criteria, the conventional culture-based method has limitations, and definitive treatment is often delayed (10).

MRI, CT, and ultrasound are useful for the diagnosis of CDC. However, these imaging methods do not always succeed in distinguishing fungal abscesses in the active phase from possible (even if rare) bacterial and tubercular causes $(11,12)$.

With the development of molecular-biology methods, diagnosis using nucleic acids in tissue was introduced in the 2020 European Organization for Research and Treatment of Cancer/Mycoses Study Group guideline of invasive fungal disease (6) compared with recommendations from the 2008 version (4). The guideline of 2020 version (6) recommended each of the following investigations as essential for the diagnosis of CDC: microscopic analyses from sterile material (including histopathologic, cytopathologic, or direct microscopic examination of a specimen); culture from sterile material; blood culture; nucleic-acid diagnosis from tissue.

A conventional method of direct microscopic examination, $\mathrm{CFW}$, was introduced to the diagnosis of CDC. The rationale of CFW staining is to detect (non-specifically) $\beta(1-3)$ and $\beta(1-4)$ polysaccharides of the chitin-rich areas found in the cell walls of Candida species and most other fungi (13). Also, addition of CFW to preparations of potassium hydroxide can improve detection of cells from Candida species by increasing the contrast from background debris (14). Compared with time-consuming conventional cultures, fluorescent staining of biopsy tissues requires only $\leq 1 \mathrm{~min}$ for specimen generation and $\sim 10 \mathrm{~min}$ to read samples, and is a more economical option (15). However, the disadvantage of CFW is the lack of ability to identify the species of fungus.

mNGS was an emerging culture-independent, highthroughput, microbiological diagnostic approach (16). It is relatively effective in cataloging and detecting pathogens, especially for uncommon or non-cultivable species (17). mNGS offers a faster and less biased non-culture-based methodology of pathogen detection through sequencing of extracted DNA from the sample directly (18). Therefore, mNGS has been applied to detect causal pathogens in deep-seated tissue samples $(18,19)$, such as in tissue from the lungs, (20), liver (21), brain (22), and cardiac-valve vegetation (23). We reported, for the first time, CDC diagnosed by mNGS. In this way, we showed that mNGS is a fast and non-culture-dependent diagnostic method.

The duration of CDC treatment can be 3-6 months (24). Usually, fluconazole is chosen after induction by CAP or AmB (25). With the escalating resistance of Candida species to azole (26-28), identification of Candida species has become important for the antifungal strategy in CDC patients. For the C. tropicalis, it appeared to be sensitive to fluconazole. However, drugsusceptibility testing of 20,788 strains of Candida species from 39 countries showed increased resistance to fluconazole for
C. tropicalis of $2.5 \%$ in 1997 to $4.9 \%$ in 2014 (28). A survey of patients in intensive care units in China showed that the prevalence of resistance of $C$. tropicalis to fluconazole was $19.3 \%$, but there was no resistance to CAP or AmB (29). Pathogen identification is becoming increasingly important in culturenegative CDC patients, and mNGS is a good choice.

Patients with CDC appear to occur after recovering from neutropenia, which suggests that $\mathrm{CDC}$ is a manifestation of immune reconstitution inflammatory syndrome (IRIS). Compared with use of an antifungal agent alone, adjuvant therapy using corticosteroids for a short course and of medium dose could benefit for patients with CDC by hastening improvement of clinical symptoms (30). Therefore, if empirical use of antifungal agents is not efficacious, identification of the pathogen in CDC is crucial to distinguish if it is caused by drug resistance or IRIS. Hence, development and validation of nonculture diagnostic methods for CDC is a top medical priority.

We combined the results from mNGS with those of CFW staining from liver biopsies. This strategy enabled confirmation of the diagnosis of CDC and guided antifungal treatment and subsequent adjuvant therapy using the corticosteroid DXM.

The histopathology manifestations of CDC are associated with three hepatic patterns: necrosis, abscess, and granuloma (31). Acute histopathology is manifested as a minimal inflammatory reaction in the neutropenic stage, whereas microabscesses with an intense inflammatory reaction occur after recovery from neutropenia. The budding cells of Candida species with pseudohyphae or hyphae can be seen in the center of necrotic tissue. Chronic histopathology manifests as granulomatous lesions. These are characterized by fibrosis or central necrosis surrounded by giant-cell granulomatous tissue with fibroblasts and/or macrophages arranged in palisade (9). There were only a few budding cells of Candida species with pseudohyphae or hyphae in the lesions in our patient. Therefore, when carrying out ultrasound-guided liver biopsy, the needle should reach the lesion center to have a greater chance of obtaining the pathogen. This strategy also explains why this specific histology feature was detected in the second liver biopsy but not in the first liver biopsy.

A combination of mNGS and CFW staining may be an alternative option for a culture-negative diagnosis of fungal infection. Nevertheless, the absence of detection of antibiotic resistance by mNGS was a limitation in this case study.

\section{CONCLUSIONS}

mNGS testing of a liver-biopsy sample along with CFW staining is a fast and reliable method for obtaining the proven diagnosis of culture-negative CDC. Early identification of the infective pathogens in culture-negative CDC can guide pathogen-targeted antifungal chemotherapy and subsequent adjuvant therapy with corticosteroids and/or splenectomy if indicated.

\section{DATA AVAILABILITY STATEMENT}

The raw data supporting the conclusions of this article will be made available by the authors, without undue reservation. 


\section{ETHICS STATEMENT}

The studies involving human participants were reviewed and approved by Clinical Research Ethics Committee of the First Affiliated Hospital, Zhejiang University School of Medicine. The patient provided the written informed consent to participate in this study. Written informed consent was obtained from the patient for the publication of any potentially identifiable images or data included in this article.

\section{AUTHOR CONTRIBUTIONS}

YZ designed research. YJ, ZW, and YZ analyzed and collected data, and wrote the manuscript. CZ, BH, XW, QY, YL, and $\mathrm{XY}$ collected clinical data. All authors read and approved the final manuscript.

\section{REFERENCES}

1. Sallah S, Semelka RC, Wehbie R, Sallah W, Nguyen NP, Vos P. Hepatosplenic candidiasis in patients with acute leukaemia. Br J Haematol. (1999) 106:697701. doi: 10.1046/j.1365-2141.1999.01592.x

2. Anttila VJ, Elonen E, Nordling S, Sivonen A, Ruutu T, Ruutu P. Hepatosplenic candidiasis in patients with acute leukemia: incidence and prognostic implications. Clin Infect Dis. (1997) 24:375-80. doi: 10.1093/clinids/24.3.375

3. Pagano L, Mele L, Fianchi L, Melillo L, Martino B, D’Antonio D, et al. Chronic disseminated candidiasis in patients with hematologic malignancies. Clinical features and outcome of 29 episodes. Haematologica. (2002) 87:53541. doi: 10.1016/S0301-472X(02)00785-3

4. De Pauw B, Walsh TJ, Donnelly JP, Stevens DA, Edwards JE, Calandra T, et al. Revised definitions of invasive fungal disease from the European Organization for Research and Treatment of Cancer/Invasive Fungal Infections Cooperative Group and the National Institute of Allergy and Infectious Diseases Mycoses Study Group (EORTC/MSG) Consensus Group. Clin Infect Dis. (2008) 46:1813-21. doi: 10.1086/588660

5. Masood A, Sallah S. Chronic disseminated candidiasis in patients with acute leukemia: emphasis on diagnostic definition and treatment. Leuk Res. (2005) 29:493-501. doi: 10.1016/j.leukres.2004.10.003

6. Donnelly JP, Chen SC, Kauffman CA, Steinbach WJ, Baddley JW, Verweij PE, et al. Revision and update of the Consensus Definitions of Invasive Fungal Disease From the European Organization for Research and Treatment of Cancer and the Mycoses Study Group Education and Research Consortium. Clin Infect Dis. (2020) 71:1367-76. doi: 10.1093/cid/ciz1008

7. Chen CY, Cheng A, Tien FM, Lee PC, Tien HF, Sheng WH, et al. Chronic disseminated candidiasis manifesting as hepatosplenic abscesses among patients with hematological malignancies. BMC Infect Dis. (2019) 19:635. doi: 10.1186/s12879-019-4260-4

8. Anttila VJ, Ruutu P, Bondestam S, Jansson SE, Nordling S, Farkkila M, et al. Hepatosplenic yeast infection in patients with acute leukemia: a diagnostic problem. Clin Infect Dis. (1994) 18:979-81. doi: 10.1093/clinids/18.6.979

9. Kontoyiannis DP, Luna MA, Samuels BI, Bodey GP. Hepatosplenic candidiasis. A manifestation of chronic disseminated candidiasis. Infect Dis Clin North Am. (2000) 14:721-39. doi: 10.1016/S0891-5520(05)70128-8

10. Clancy CJ, Nguyen MH. Finding the "missing 50\%" of invasive candidiasis: how nonculture diagnostics will improve understanding of disease spectrum and transform patient care. Clin Infect Dis. (2013) 56:1284-92. doi: $10.1093 / \mathrm{cid} / \mathrm{cit} 006$

11. Pagano L, Larocca LM, Marra R, Pizzigallo E, Leone G. A leukemic patient with hepatosplenic abscesses due to coagulase-negative staphylococci. Clin Infect Dis. (1992) 14:364-5. doi: 10.1093/clinids/14.1.364

12. Lee DG, Choi JH, Kim YJ, Lee S, Min CK, Kim DW, et al. Hepatosplenic tuberculosis mimicking disseminated candidiasis in patients with acute leukemia. Int J Hematol. (2001) 73:119-21. doi: 10.1007/BF02981913

\section{FUNDING}

This work was supported by Fundamental Research Funds for the Central Universities (2019FZA7001), Zhejiang Education Department 2020 Special Project Against COVID19 (Zhejiang University 94), China National Science and Technology Major Project (2017ZX10202202), Youth Fund Project of Zhejiang Provincial Natural Science Foundation, (LQ17H030002), and Haining Science and Technology Project (201805).

\section{ACKNOWLEDGMENTS}

We thank Dr. Dewi Andayani for her critical language editing.

13. Pringle JR, Preston RA, Adams AE, Stearns T, Drubin DG, Haarer BK, et al. Fluorescence microscopy methods for yeast. Methods Cell Biol. (1989) 31:357-435. doi: 10.1016/s0091-679x(08)61620-9

14. Perry JL, Miller GR. Quality control slide for potassium hydroxide and cellufluor fungal preparations. J Clin Microbiol. (1989) 27:1411-2.

15. Harrington BJ, Hageage GJ. Calcofluor white: a review of its uses and applications in clinical mycology and parasitology. Lab Med. (2003) 34:361-7. doi: 10.1309/eph2tdt8335gh0r3

16. Lefterova MI, Suarez CJ, Banaei N, Pinsky BA. Next-generation sequencing for infectious disease diagnosis and management: a report of the Association for Molecular Pathology. J Mol Diagn. (2015) 17:623-34. doi: 10.1016/j.jmoldx.2015.07.004

17. Besser J, Carleton HA, Gerner-Smidt P, Lindsey RL, Trees E. Nextgeneration sequencing technologies and their application to the study and control of bacterial infections. Clin Microbiol Infect. (2018) 24:335-41. doi: 10.1016/j.cmi.2017.10.013

18. Miao Q, Ma Y, Wang Q, Pan J, Zhang Y, Jin W, et al. Microbiological diagnostic performance of metagenomic next-generation sequencing when applied to clinical practice. Clin Infect Dis. (2018) 67:S231-40. doi: $10.1093 / \mathrm{cid} /$ ciy693

19. Zhang HC, Ai JW, Cui P, Zhu YM, Li YJ, Zhang WH. Incremental value of metagenomic next generation sequencing for the diagnosis of suspected focal infection in adults. J Infect. (2019) 79:419-25. doi: 10.1016/j.jinf.2019. 08.012

20. Li H, Gao H, Meng H, Wang Q, Li S, Chen H, et al. Detection of pulmonary infectious pathogens from lung biopsy tissues by metagenomic next-generation sequencing. Front Cell Infect Microbiol. (2018) 8:205. doi: 10.3389/fcimb.2018.00205

21. Ai JW, Li Y, Cheng Q, Cui P, Wu HL, Xu B, et al. Diagnosis of local hepatic tuberculosis through next-generation sequencing: smarter, faster and better. Clin Res Hepatol Gastroenterol. (2018) 42:178-81. doi: 10.1016/j.clinre.2018.04.007

22. Salzberg SL, Breitwieser FP, Kumar A, Hao H, Burger P, Rodriguez FJ, et al. Next-generation sequencing in neuropathologic diagnosis of infections of the nervous system. Neurol Neuroimmunol Neuroinflamm. (2016) 3:e251. doi: 10.1212/nxi.0000000000000251

23. Fukui Y, Aoki K, Okuma S, Sato T, Ishii Y, Tateda K. Metagenomic analysis for detecting pathogens in culture-negative infective endocarditis. $J$ Infect Chemother. (2015) 21:882-4. doi: 10.1016/j.jiac.2015.08.007

24. Pappas PG, Rex JH, Sobel JD, Filler SG, Dismukes WE, Walsh TJ, et al. Guidelines for treatment of candidiasis. Clin Infect Dis. (2004) 38:161-89. doi: $10.1086 / 380796$

25. Pappas PG, Kauffman CA, Andes DR, Clancy CJ, Marr KA, Ostrosky-Zeichner L, et al. Clinical practice guideline for the management of candidiasis: 2016 Update by the Infectious Diseases Society of America. Clin Infect Dis. (2016) 62:e1-50. doi: 10.1093/cid/civ933 
26. Pfaller MA, Diekema DJ, Gibbs DL, Newell VA, Ellis D, Tullio V, et al. Results from the ARTEMIS DISK Global Antifungal Surveillance Study, 1997 to 2007: a 10.5-year analysis of susceptibilities of Candida species to fluconazole and voriconazole as determined by CLSI standardized disk diffusion. J Clin Microbiol. (2010) 48:1366-77. doi: 10.1128/jcm.02117-09

27. Xiao M, Fan X, Chen SC, Wang H, Sun ZY, Liao K, et al. Antifungal susceptibilities of Candida glabrata species complex, Candida krusei, Candida parapsilosis species complex and Candida tropicalis causing invasive candidiasis in China: 3 year national surveillance. J Antimicrob Chemother. (2015) 70:802-10. doi: 10.1093/jac/dku460

28. Pfaller MA, Diekema DJ, Turnidge JD, Castanheira M, Jones RN. Twenty years of the SENTRY Antifungal Surveillance Program: results for Candida species from 1997-2016. Open Forum Infect Dis. (2019) 6:S79-94. doi: 10.1093/ofid/ofy358

29. Liu W, Tan J, Sun J, Xu Z, Li M, Yang Q, et al. Invasive candidiasis in intensive care units in China: in vitro antifungal susceptibility in the ChinaSCAN study. J Antimicrob Chemother. (2014) 69:162-7. doi: 10.1093/jac/d kt330
30. Legrand F, Lecuit M, Dupont B, Bellaton E, Huerre M, Rohrlich PS, et al. Adjuvant corticosteroid therapy for chronic disseminated candidiasis. Clin Infect Dis. (2008) 46:696-702. doi: 10.1086/527390

31. Rammaert B, Desjardins A, Lortholary O. New insights into hepatosplenic candidosis, a manifestation of chronic disseminated candidosis. Mycoses. (2012) 55:e74-84. doi: 10.1111/j.1439-0507.2012.02182.x

Conflict of Interest: The authors declare that the research was conducted in the absence of any commercial or financial relationships that could be construed as a potential conflict of interest.

Copyright (c) 2021 Jin, Wang, Zhu, Yang, Lu, Yu, Hong, Wang and Zhang. This is an open-access article distributed under the terms of the Creative Commons Attribution License (CC BY). The use, distribution or reproduction in other forums is permitted, provided the original author(s) and the copyright owner(s) are credited and that the original publication in this journal is cited, in accordance with accepted academic practice. No use, distribution or reproduction is permitted which does not comply with these terms. 